The Egyptian Journal of Hospital Medicine (January 2019) Vol. 74 (6), Page 1226-1232

\title{
Muscle Invasive Bladder Cancer: comparison between Radical Cystectomy and Bladder Sparing Therapy
}

\author{
Mohamed Esmat $^{* 1}$, Sabri Moussa², Wael El-Sheshtawy ${ }^{3}$ \\ Departments of ${ }^{1}$ Surgery, ${ }^{2}$ Urology and ${ }^{3}$ Clinical Oncology, Faculty of Medicine, Al-Azhar University, \\ Cairo Egypt \\ * Corresponding Author: Prof. Mohamed Esmat, E-Mail: Prof.mohamed.esmat@ gmail.com
}

\begin{abstract}
Background: bladder cancer (BC) is a life menacing disease that harvested a considerable number of deaths anniversary. Noteworthy, $\mathrm{BC}$ is the most predominant cancer among Egyptian men with a prevalence rate of more than $15 \%$ resulting in 8,000 deaths yearly. Aim of the work: this study was implemented to retrieve which modality (Radical cystectomy (RC) vs bladder-sparing therapy (BST)) had the superiority in the management of patients with muscle-invasive bladder cancer (MIBC). Methods: we performed a retrospective, case-control study from the archived files at the Urology Department in coordination with the Surgical Oncology Unit and Clinical Oncology Department at Bab-Elsharya and Al-Hussein University Hospitals, Faculty of Medicine, Al-Azhar University, Cairo between January 2007 and October 2013. Patients with MIBC, Stage II (T2a-T2b, N0, M0) or Stage III (T3a-T4a, N0, M0), and suitable for chemoradiation and surgery were enrolled in this study. Results: this study comprehended an overall 148 candidates diagnosed with MIBC. Of them, 95 participants were submitted to RC, whilst 53 patients were subordinated to BST. The overall 5-year survival rate was $58.9 \%$ and $39.6 \%$ in RC and BST groups, respectively (log-rank test, $\mathrm{P}=0.273$ ). Additionally, disease-free survival rates were $86.8 \%$ in patients subjected to BST and $91.6 \%$ in patients subjected to RC (log-rank test, $\mathrm{p}=0.6$ ). Conclusion: despite no differences were established between RC and BST regarding overall survival rate and disease-free survival rate, BST is an emerging procedure with considerable low complications relative to RC.
\end{abstract}

Keywords: bladder-sparing therapy, muscle-invasive bladder cancer, radical-cystectomy.

\section{Introduction}

Bladder cancer $(\mathrm{BC})$ is a life menacing disease that harvested a considerable number of deaths anniversary. Noteworthy, BC is the most predominant cancer among Egyptian men with a prevalence rate of more than $15 \%$ resulting in 8,000 deaths yearly ${ }^{(1,2)}$. Transitional cell carcinoma (TCC), which is related mainly to tobacco smoking, is the main type of $\mathrm{BC}$ with an incidence rate of more than $90 \%{ }^{(3)}$. Of note, Muscle-invasive bladder cancer (MIBC) constituted about $25 \%$ among patients with $\mathrm{BC}^{(4)}$. Herein, a high concern should be given to MIBC patients particularly with the obscurity of the adequate treatment. Throughout the past decade, radical cystectomy (RC) approach was the standard treatment of patients with $\mathrm{MIBC}^{(5)}$. However, this approach was associated with conceivable morbidities that ameliorate the patient's quality of life and leads to a high mortality rate principally in old age patients ${ }^{(\boldsymbol{6})}$. Thereafter, to ensure the patients quality of life and the oncological outcomes, the current tendency in the management of tumors focused on preserving the affected organs with subsequent therapy based on chemotherapy and radiotherapy ${ }^{(7)}$. Currently, bladder-sparing therapy (BST), based on trans-urethral resection of bladder tumor (TURBT) followed by radiotherapy and chemotherapy, is a promising approach in the management of patients with BC with comparatively concise advantages in the terms of maintaining bladder function, achieving oncological outcomes, and considerably lower rats of morbidities and mortalities ${ }^{(8,}{ }^{9}$ ). The optimal management of patients with MIBC is still a doubtful question in the literature especially with the diversity in the surgical techniques, treatment modalities and patient's demographic characteristics. Thereafter, this study was implemented to retrieve which modality (RC vs BST) had the superiority in the management of patients with MIBC apart from 
oncological outcomes, complications, and patient's quality of life.

\section{Methods}

\section{Ethical approval}

This study was confirmed and the IRB was obtained from the ethics unite of the Faculty of Medicine, Al-Azhar University. The informed agreements were gained from the included participants with consideration to the Declaration of Helsinki.

\section{Study design}

We performed a retrospective, case-control study from the archived files at the Urology department in coordination with the Surgical Oncology Unit and Clinical Oncology Department at BabElsharya and Al-Hussein University Hospitals, Faculty of Medicine, Al-Azhar University, Cairo between January 2007 and October 2013.

\section{Study population}

A total of 148 participants diagnosed with BC were enrolled in the study. Of them, 95 patients were subjected to RC whilst 53 patients were subjected to BST

\section{Eligibility criteria}

Patients with MIBC, Stage II (T2a-T2b, N0, M0) or Stage III (T3a-T4a, N0, M0), and suitable for chemo-radiation and surgery; therefore, they were treated either by BST or RC. Otherwise, we omitted patients with stage I or stage IVb and patients received RC with radiotherapy or chemotherapy.

\section{Data collection}

The medical files of participants enrolled in this study were subordinated to rigorous revision and the following data were collected, age, sex, BMI, marital status and associated co-morbidities. Subsequently, the clinical examination included the vital signs, DRE of the tumor, DRE of the prostate, the state of the anal sphincter and pervaginal examination in the females. Routinepreoperative investigations included blood profile, liver function tests and kidney function tests were performed. Moreover, intravenousurography (IVU) and abdominal-pelvic ultrasound were reviewed for further assessment of the kidney function and the tumor. All participants data were undergone to quality appreciation and revised by a surgical oncologist, urologist, and clinical oncologist.

\section{Tumor diagnosis and staging}

The clinical staging of the candidates was performed in conformity with the TNM-system (10); counterpart tumor (T) staging was assessed by bimanual palpation of the tumor, pre and after TURBT, also tumor invasion depth was appreciated histologically or after RC. lymph node $(\mathrm{N})$ staging was determined by $\mathrm{CT}$-scan or nodal dissection. Tumor metastasis $(\mathrm{M})$ were evaluated by using chest $\mathrm{x}$-ray, abdominal-pelvic ultrasound, and CT-scan. Specimens were obtained by TURBT or samples from the bladder in patients with suspicious BC. Histopathological examination and histological grading of the tumor was estimated in accordance with the World Health Organization and International Society of Urological Pathology ${ }^{(\mathbf{1 1})}$.

\section{Surgical procedure}

Radical cystectomy was exemplified as total removal of the urinary bladder together with or without pelvic lymphadenectomy, removal of the obturator lymph nodes and iliac lymph nodes to the range of mid-common iliac vessels or close organs extirpation as the distal end of the ureter in addition to prostate along with seminal vesicles in males and uterus, ovaries, along with portion of the vagina in females. Subsequently, bladder reconstruction was accomplished with urinary diversion based on the patients' status and the evaluation of the surgeon.

\section{Bladder-sparing therapy}

The BST institutional protocol was to start three dimensional radiotherapy (after maximum TURBT) to the whole pelvis (bladder and pelvic lymph nodes) to a dose between 45-50 Gy followed by a boost on the bladder to reach a total dose of 60-66 Gy over 6-7 weeks of treatment, concurrent with platinum-based chemotherapy either cisplatin or carboplatin (for patients with impaired renal functions) on weekly bases as a single agent or in combination with either weekly paclitaxel or gemcitabine.

\section{Follow up}

To retrieve any unexpected adverse events, all patients were submitted to close follow up period with a median follows up of among patients treated with RC of 14 (3-60) months, whilst the median follows up of the patients received BST 
was 13.5 (3-60) months. The follow-up protocol comprehended a regular physical examination, radiological evaluation, cystoscope, and ultrasonography of the abdomen and pelvis. Patients with preserved bladder were submitted to further evaluation to detect the incomplete clinical response.

\section{Statistical analysis}

Normally- distributed data were notified using mean and standard deviation (SD) and the particular groups were compared by using students t-test. Non-parametric variables were expressed as median and range, and the difference between those groups was estimated using Mann-Whitney $U$ test. Moreover, categorical variables were elucidated as number and percentage and compared using chi-square test. Survival analysis was done using KaplanMeier methods to calculate 5-year survival rate and disease-free survival rate. The significant was established when $\mathrm{p}<0.05$. Analyses was performed using SPSS v.23 software (IBM SPSS Statistics; IBM Corp, Armonk, NY, USA), and e of SCC and adenocarcinoma (Table 2).
MedCalc software version 14.8 (MedCalc Software, Mariakerke, Belgium). Figures were renovated using GraphPad Prism (GraphPad Software, Inc, San Diego) software version 7 .

\section{Results}

\section{Patient's demographic characteristics}

This study comprehended an overall 148 candidates diagnosed with MIBC. Of them, 95 participants were submitted to RC whilst 53 patients were subordinated to BST. Patients' mean of age was $56.34 \pm 8.64$ years for RC group while it was $59.07 \pm 8.85$ years for BST group. No significant difference was observed between both groups in terms of patient's characteristics except for kidney functions and imaging results and anaemia (Table 1). TCC was the main pathology, $62(65.3 \%)$ patients within RC group and all patients within the BST group, and superior to the incidence.

Table 1. Patient's demographic characteristics

\begin{tabular}{|c|c|c|c|}
\hline & $\mathrm{RC}=95$ & BST $=53$ & p-value \\
\hline & Mean (SD)/ N (\%) & Mean (SD)/ N (\%) & \\
\hline Age & $56.34(8.64)$ & $59.07(8.85)$ & 0.07 \\
\hline Male & $70(73.7)$ & $43(81.1)$ & 0.42 \\
\hline History of smoking & $60(63.2)$ & $31(58.5)$ & 0.143 \\
\hline \multicolumn{4}{|l|}{ Co-morbidities } \\
\hline Hypertension & $25(26.3)$ & $10(18,9)$ & 0.42 \\
\hline Diabetes & $7(7.4)$ & $6(11.3)$ & 0.546 \\
\hline Bilharziasis & $10(10.5)$ & $5(9.4)$ & 0.34 \\
\hline Cardiac insufficiency & $0(0)$ & $2(3.8)$ & - \\
\hline Chronic kidney disease & $0(0)$ & $2(3.8)$ & - \\
\hline DVT & 0 & $1(1.9)$ & - \\
\hline Elevated liver function tests & $90(94.7)$ & $49(92.5)$ & 0.577 \\
\hline Anemia & $13(13.7)$ & $17(32.1)$ & 0.008 \\
\hline Kidney functions and imaging & & & 0.031 \\
\hline $\begin{array}{l}\text { Nicotinamide adenine } \\
\text { dinucleotide elevation }\end{array}$ & $93(97.9)$ & $46(86.8)$ & 0.006 \\
\hline Stone kidney & $2(2.1)$ & $5(9.4)$ & .044 \\
\hline Stone bladder & 0 & $2(3.8)$ & - \\
\hline
\end{tabular}




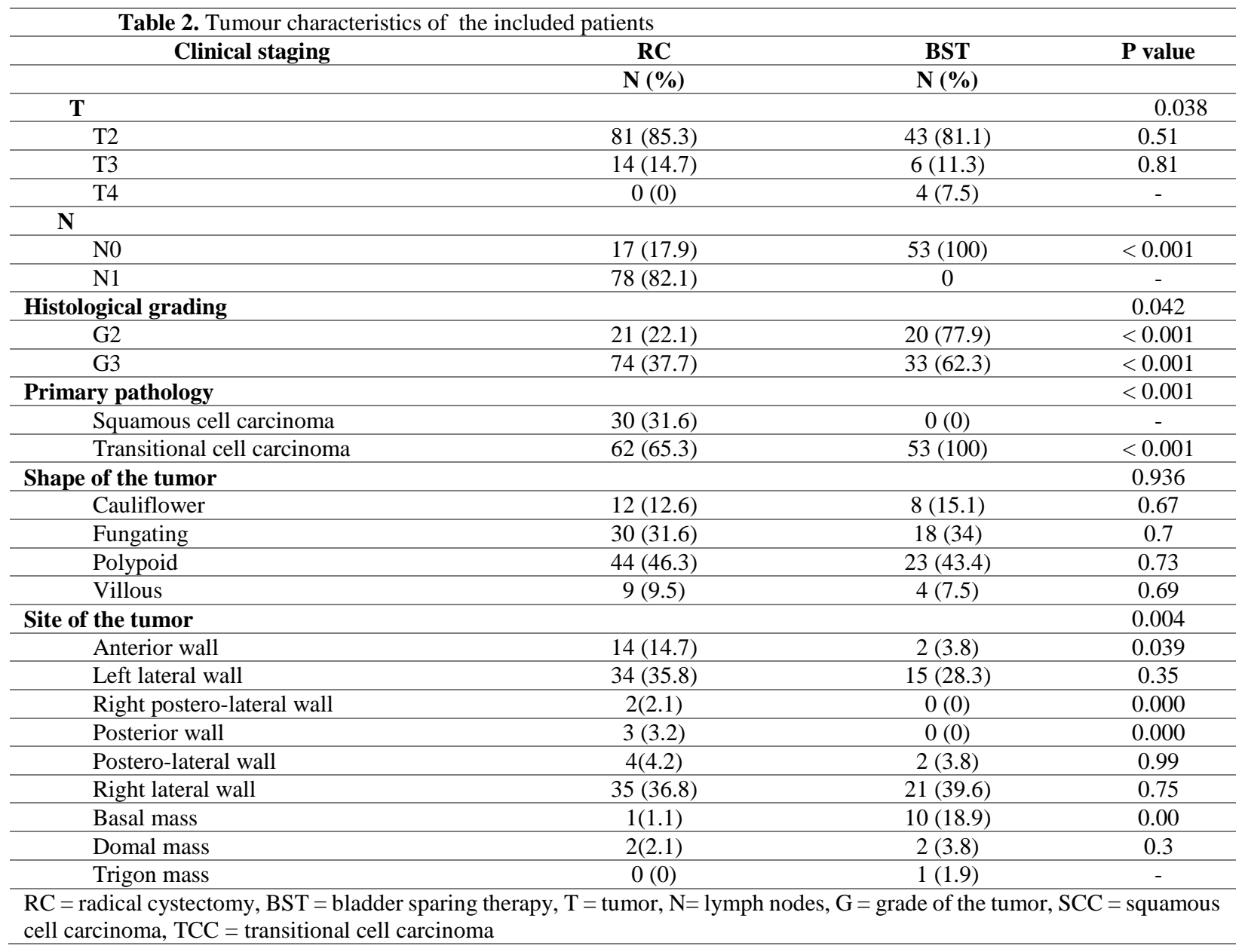

In RC group, bladder reconstruction using ileal conduit was used in 28 patients whilst, sigmoid pouch and ileal neobladder were used in 27 and 24 patients, respectively. Regarding postoperative complications, $72(77.4 \%)$ patients treated by RC experienced fever as the main early post-operative complications. Moreover, in RC group, 25 (26.3\%) patients suffered from wound infection as a post-operative complication. Furthermore, 14 out the 53 patients who had treated with BST developed GIII-IV toxicity, the main toxicities were urine frequency and dysuria in 16 patients, neutropenia in 10 patients, and proctitis in 9 patients. Deterioration of the renal function was reported in 9 patients, this renal impairment mandate discontinuation of treatment in 4 patients. Throughout the study period, 39 $(41.05 \%)$ patients died within the RC group whilst $32(60.3 \%)$ patients died within the BST group with a significant difference between the two groups $(P=0.002)$. Furthermore, the main cause of death among RC group was septicaemia, $14(35.8 \%)$ patients, followed by disease recurrence, $11(28.8 \%)$ patients, which was the main cause of death in BST group,23 $(71.8 \%)$ patients, with significant difference between the both modalities $(\mathrm{P}<0.005)$. The overall 5-year survival rate was $58.9 \%$ and $39.6 \%$ in $\mathrm{RC}$ and BST groups, respectively (log-rank test, $\mathrm{P}=$ 0.273) (Fig. 1A). Additionally, disease-free survival rates were $86.8 \%$ in patients subjected to BST and $91.6 \%$ in patients subjected to RC (logrank test, $p=0.6$ ). (Fig. 1B). Having the T2 stage, the 5-years survival rate of patients was $57.9 \%$ and $41.9 \%$ in the RC and BST groups, respectively (log-rank test, $\mathrm{P}=0.038$ ) (Figure 1C). The disease-free survival rate of patients with the clinical T2 stage was $91.3 \%$ in patients received $\mathrm{RC}$ and $88.4 \%$ in patients treated with BST (log-rank test, $\mathrm{P}=0.63$ ) (Fig. 1D). 

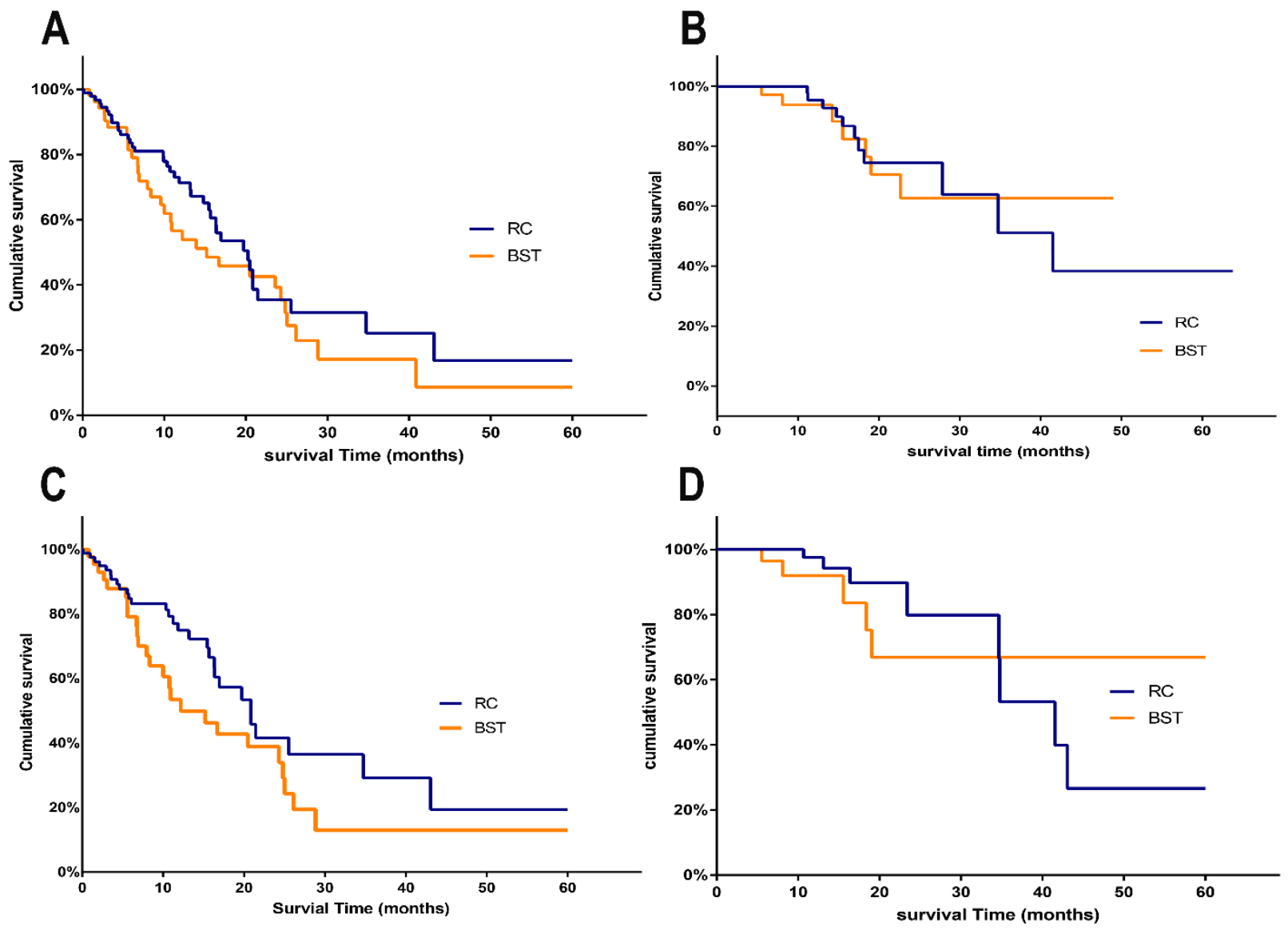

Figure.1 Kaplan-Meier curves compared between RC and BST groups regarding as the overall 5-year survival(A) and the 5-year disease-free survival rate (B). panel (A) showed that overall survival rate for all patients in the two groups was $58.9 \%$ and $39.6 \%$ in RC and BST groups, respectively. Panel (B) showed that the 5-year recurrence free survival was $90.6 \%$ and $86.8 \%$ in RC and BST groups, respectively. (C) Overall 5-year survival rate among patients with clinical T2 stage within RC and BST groups (D) Overall 5-year disease-free survival rate among patients with clinical T2 stage within RC and BST groups.

\section{Discussion}

MIBC is a lethal disease with a survival rate of $15 \%$ in patients with TCC if left untreated. Herein, aggressive therapy with potential adequacy in the achieving of the oncological and surgical outcomes is reacquired. This study shed the light on the best approach in the treatment of patients with $\mathrm{MIBC}^{\left(\mathbf{3},{ }^{12}\right)}$. The evidence summarized in our study brings to light that BST was emerging and promising approach in patients with MIBC apart from the similar overallsurvival rate and disease-free survival rate with a considerable low rate of complications and better quality of life. However, RC achieved notability in patients with $\mathrm{T} 2$ stage regarding overall survival rate.

In accordance with our results, Munro et al. ${ }^{(13)}$ notified the obscurity of substantially significant difference between RC and BST not only regarding 5-years survival rate but also regarding 10 years survival rate. Supit et al. ${ }^{(14)}$ compared RC with TURBT plus radiotherapy and showed no difference between the two groups regarding the 5-year overall survival rate (log-rank test, 
$\mathrm{P}=0.118$ ). Similarly, a systematic review and meta-analysis, that enrolled 9554 participants, reported no distinction regarding the 5-year overall survival rates between both modalities (log-rank, $\mathrm{p}=0.778) .{ }^{(15)}$ Consequently, within five years, the recurrence rate among patients treated by RC was $11.6 \%$ whilst, it was $13.2 \%$ in patients received BST. Conversely, Algizawy et al. (16) showed a high recurrence rate among patients submitted to RC relative to BST (28.8\% vs $24.2 \%$ ). This discrepancy may be evolved due to the significant diversity of the patient's demographic, and tumor characteristics between both studies particularly with a significant portion of tumor multifocality, $14(17.5 \%)$ patients in Algizawy et al. (16) study. In our study, only 5 (9.4\%) patients within BST had bilharziasis, whilst no patient had tumor multifocality. Moreover, most of the patients received RC was a clinical T2 stage (85.3\%), with a single tumor mass (94.7\%). Contrarily, in Algizawy et al. study, most of the patients received BST had bilharziasis, $60(75 \%)$ patients, with a higher proportion of tumor multifocality, 14 (17.5\%) patients. Within three years prior to $\mathrm{RC}$, the majority of patients died from the complications of the surgery, not from BC itself ${ }^{(17)}$. Herein, the considerable percentage of complications among patients subjected to RC in our study showed that a high concern should be given to these patients to avoid the subsequent mortality. Having the tumor stage into consideration, our results showed that RC was more suitable for patients with T2 stage regarding the 5-years overall survival rate. This can be explained through the more aggressive nature of the tumor in such stage that makes BST not appropriate for patients with T2 stage or more. ${ }^{(\mathbf{1 4})}$ This discrepancy that favor $\mathrm{RC}$ over BST has based on not only the tumor stage, but also the age of the patients ${ }^{(18)}$

The evidence retrieved in our study may be altered by several limitations such as the retrospective nature of the study, the limited sample size that hinder the accessibility of subgroup analysis for clinical T3 patients, Moreover, the heterogeneity between the included candidates regarding demographic and tumor characteristics may limit the power of the study. Herein, a multicenter randomized clinical trial is mandatory to overcome the limitations of the study.

\section{Conclusions}

The result of this study brings to light that despite no difference were established between RC and BST regarding overall survival rate and diseasefree survival rate, BST is an emerging procedure with considerable low complications relative to $\mathrm{RC}$.

\section{References}

1.Ibrahim AS, Khaled HM, Mikhail NN, Baraka $H$ and Kamel H(2014): Cancer incidence in Egypt: results of the national population-based cancer registry program. Journal of cancer epidemiology, 134(2):381-395. 2.Pashootan P, Legrand $G$ and Ploussard $G$ (2014): Muscle-invasive bladder cancer. La Revue du Praticien, 64(10): 1391-1398.

3.Chou R, Selph SS, Buckley DI, Gustafson KS, Griffin JC, Grusing SE et al.(2016): Treatment of muscle-invasive bladder cancer: A systematic review. Cancer,122(6) 842-851.

4.Ferlay J, Steliarova-Foucher E, LortetTieulent J, Rosso S, Coebergh J-WW, Comber H et al.(2013): Cancer incidence and mortality patterns in Europe: estimates for 40 countries in 2012. European journal of cancer,49(6) 13741403.

5.Tsao CK, Liaw BC, Oh WK and Galsky MD(2016): Muscle invasive bladder cancer closing the gap between practice and evidence. The Italian Journal of Urology and Nephrology, 67(1) :65-73.

6.Rose TL and Milowsky MI (2015): Management of muscle-invasive bladder cancer in the elderly.Current Opinion in Urology,25(5): 459-467.

7.Krishna SR and Konety BR (2017): Current concepts in the management of muscle invasive bladder cancer. Indian Journal of Surgical Oncology,8(1): 74-81.

8.Mathes $J$, Rausch $S$, Todenhofer $T$ and Stenzl A (2018): Trimodal therapy for muscleinvasive bladder cancer. Expert Review of Anticancer Therapy,18(12): 1219-1229.

9.Chedgy EC and Black PC(2016): Radical Cystectomy and the Multidisciplinary 
Management of Muscle-Invasive Bladder Cancer. JAMA oncology,2(7): 855-6.

10.Singletary SE and Greene FL(2003): Revision of breast cancer staging: the 6th edition of the TNM Classification. Seminars in surgical oncology, 17(1): 346-1.

11.Epstein JI, Amin MB, Reuter VR, Mostofi FK (1998): Neoplasms of the urinary bladder. The American journal of surgical pathology,22(12): 1435-48.

12.Skinner EC (2018): Treatment of MuscleInvasive Bladder Cancer in Older Patients. ascopubs.org/doi/full/10.1200/EDBK_158974

13.Munro NP, Sundaram SK, Weston PM, Fairley L, Harrison SC, Forman D et al.(2010):

A 10-year retrospective review of a nonrandomized cohort of 458 patients undergoing radical radiotherapy or cystectomy in Yorkshire, UK. International Journal of Radiation Oncology* Biology* Physics,77(1): 119-24.

14.Supit W, Mochtar CA, Santoso RB and Umbas R (2014): Outcomes of radical cystectomy and bladder preservation treatment for muscle-invasive urothelial carcinoma of the bladder. Asian journal of surgery,37(4): 184-9.
15.Vashistha V, Wang H, Mazzone A, Liss MA, Svatek RS, Schleicher M et al.(2017): Radical cystectomy compared to combined modality treatment for muscle-invasive bladder cancer: A systematic review and meta-analysis. International Journal of Radiation Oncology* Biology* Physics,97(5): 1002-20.

16.AlGizawy SM, Essa HH, Abdel-Wanis ME and Raheem AMA(2014): Trimodality bladdersparing approach versus radical cystectomy for invasive bladder cancer. Journal of Radiotherapy in Practice,13(4): 428-37.

17.Haque W, Verma V, Butler EB and Teh BS (2017): Radical Cystectomy Versus Chemoradiation for Muscle-invasive Bladder Cancer: Impact of Treatment Facility and Sociodemographics. Anticancer research,37(10): 5603-8.

18.Mueller J, Schrader AJ, Schrader M, Schnoeller $T$ and Jentzmik F(2013): Management of muscle-invasive bladder cancer. The Italian journal of urology and nephrology, 65(4): 235-48. 